\title{
Spin coherent manipulation in Josephson weak links
}

\author{
Javier Cerrillo $\odot,{ }^{1,2,{ }^{*}}$ M. Hays,${ }^{3}$ V. Fatemi $\odot,{ }^{3}$ and Alfredo Levy Yeyati $\odot^{2,4, \uparrow}$ \\ ${ }^{1}$ Área de Física Aplicada, Universidad Politécnica de Cartagena, E-30202 Cartagena, Spain \\ ${ }^{2}$ Departamento de Física Teórica de la Materia Condensada C-V, Universidad Autónoma de Madrid, E-28049 Madrid, Spain \\ ${ }^{3}$ Department of Applied Physics, Yale University, New Haven, Connecticut 06520, USA \\ ${ }^{4}$ Condensed Matter Physics Center (IFIMAC) and Instituto Nicolás Cabrera, Universidad Autónoma de Madrid, E-28049 Madrid, Spain
}

(Received 25 January 2021; revised 7 April 2021; accepted 9 April 2021; published 11 May 2021)

\begin{abstract}
Novel designs of Josephson weak links based on semiconducting nanowires combined with circuit QED techniques have enabled the resolution of their fine structure due to spin-orbit interactions, opening a path towards Andreev spin qubits. Nevertheless, direct manipulation of the spin within a given Andreev state is in general suppressed compared to interdoublet manipulation in the absence of Zeeman effects. In addition, noisy spin-flip mechanisms limit any coherent manipulation protocol to spin postselection. We propose a combination of a spin polarization protocol analogous to sideband cooling with stimulated Raman adiabatic passage specifically tailored for these systems. We show this approach is robust for a large range of design parameters, including the currently rather stringent coherence times.
\end{abstract}

DOI: 10.1103/PhysRevResearch.3.L022012

While conventional superconducting qubits are becoming a mature technology [1], other radically new approaches involving superconducting nanostructures remain to be explored [2]. In particular some qubit proposals rely on the Andreev bound states (ABS) which appear in highly transmissive fewchannel Josephson weak links [3-8]. Within these proposals one can distinguish those operating when the ABS are populated with an even number of particles (the even sector), which are called Andreev level qubits (ALQ) [3-5], and the ones in which an extra unpaired quasiparticle is trapped in the weak link, which corresponds to the so-called Andreev spin qubits (ASQ) [6-8]. This type of system combines interesting aspects of microscopic degrees of freedom (such as their isolation from the environment) with the potential scalability of solid state devices. Another attractive aspect of ASQ is their inherently fermionic character and their connection to Majorana physics [9]. In fact, under sufficiently large magnetic fields the proximitized nanowires used in ASQ experiments could host Majorana states at their edges $[10,11]$.

Recent experiments on weak links formed by semiconducting nanowires coupled to a superconducting resonator in a circuit QED geometry have enabled detection of their ABS spectrum $[12,13]$, revealed their fine structure caused by spinorbit interactions [14], and offered the possibility to perform time resolved detection of their population dynamics [15]. While these results constitute a significant step forward, these

\footnotetext{
*javier.cerrillo@upct.es

†a.1.yeyati@uam.es

Published by the American Physical Society under the terms of the Creative Commons Attribution 4.0 International license. Further distribution of this work must maintain attribution to the author(s) and the published article's title, journal citation, and DOI.
}

systems still have limited controllability and, more specifically, direct spin flip transitions can be hardly implemented in the absence of magnetic fields [8]. Thus the coherent manipulation of the spin of a single quasiparticle trapped within these junctions requires special techniques which are still to be developed in the context of superconducting nanodevices.

In the present Letter, we propose to address these problems by adapting techniques which are common in the field of quantum optics. A useful protocol for the control of population transfer between two states that lack a direct coupling is the stimulated Raman adiabatic passage (STIRAP) [16]. It consists in the application of two delayed overlapping pulses in Raman configuration with respect to a third state, as illustrated in Fig. 1(a). STIRAP is known to be robust against pulse shape modifications. This is in stark contrast with the simultaneous application of two fields in Raman configuration, which requires very accurate adjustment of the pulse area to a value of $\pi$, as well as careful adjustment of the Rabi frequency $\Omega$ of both fields to similar values. In addition, a large detuning $\Delta \gg \Omega$ is needed in order to suppress population transfer to the excited levels. This reduces the effective Rabi frequency, which translates into a transfer time that is proportional to $\frac{\Delta}{\Omega^{2}}$. This is in general a factor $\frac{\Delta}{\Omega}$ slower than STIRAP.

In the context of superconducting circuits, the STIRAP protocol was first proposed for Cooper-pair boxes [17] and experimentally demonstrated on transmons [18]. Unfortunately, STIRAP techniques cannot be applied directly to the Josephson weak link case. In the devices of Refs. [14,15] with a typical junction length of the order of $400 \mathrm{~nm}$, two sets of spin-split ABSs appear within the superconducting gap. Their characteristic dispersion as a function of the superconducting phase difference $\phi$ is illustrated in Fig. 1(c). Thus, when $\phi$ is fixed to a value different from 0 or $\pi$, there are four states within the odd sector which can be connected by single 


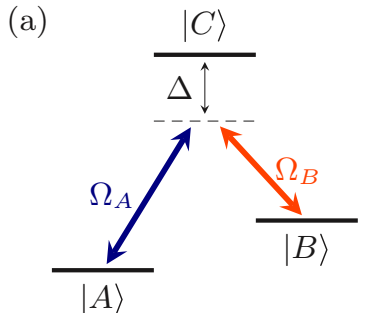

(b)

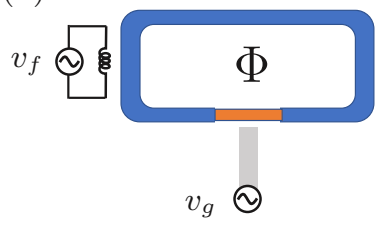

(c)

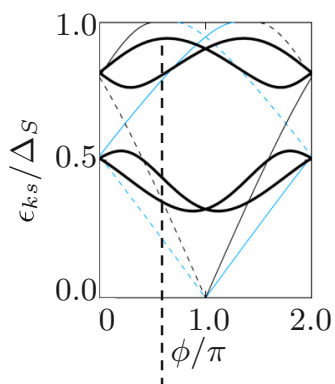

(d)

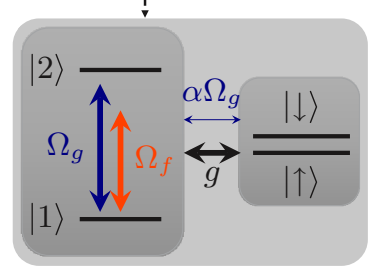

FIG. 1. (a) Traditional STIRAP setup with a three-level system in $\Lambda$ configuration and a Raman coupling generated by two fields characterized by Rabi frequencies $\Omega_{A}$ and $\Omega_{B}$. (b) Schematic diagram of the experimental setup: a superconducting loop (blue) threaded by a magnetic flux $\Phi$ is weakly linked by a semiconducting nanowire (orange). The weak link can be controlled by ac gate voltage $v_{g}$ and inductively by an ac flux voltage $v_{f}$. (c) Level dispersion relation as a function of $\phi$ for a device showing spin-split ABSs as reported in [14]. The thin lines correspond to the ballistic case and the thick lines to the presence of backscattering $(r \neq 0)$. (d) The four-level system at a fixed phase value $\phi$ as a coupled two-spin system, the first (mode) qubit represented by Pauli matrices $\tau_{i}$ and the second (spin) qubit represented by Pauli matrices $\sigma_{i}$. Gate and flux drivings induce Rabi terms $\Omega_{g} \tau_{x}$ and $\Omega_{f} \tau_{x}$, respectively. Additionally, gate driving induces a $\alpha \Omega_{g} \tau_{x} \sigma_{x}$ interaction and a ferromagnetic term $g \tau_{z} \sigma_{z}$ exists between both qubits.

particle transitions, instead of the three usually considered for STIRAP. These can be viewed as two coupled qubits, one with transition frequencies of the order of $10 \mathrm{GHz}$, which is associated with different Andreev longitudinal modes, and one with frequencies of the order of $1 \mathrm{GHz}$, which is associated with the spin degree of freedom. Ambient flux or charge noise fully mixes the spin degree of freedom [15] and thus additional spin-polarization techniques are necessary to prepare an initially pure state, which is required to apply the STIRAP protocol successfully. We also notice that intradoublet spin-flip transitions are in general suppressed compared to interdoublet transitions [8], while spin-flip transitions between manifolds are allowed with an efficiency $\alpha(\phi)$ with respect to the spin-conserving ones. This efficiency has been experimentally observed to take values in a range between 0 and 1 both for gate driving [14,19] and for flux driving $[15,20]$. Although the parameter is kept in all subsequent derivations, for simplicity in the simulations we shall assume a combination of a purely spin-conserving drive $(\alpha=0)$ as well as an $\alpha>0$ drive.

We first discuss a simplified modeling of the ABSs in these systems and then propose an implementation of a protocol analogous to STIRAP. We analyze the most appropriate preparation technique and produce an analytical model for its optimization. We show that the protocol is robust for a large range of driving strengths and spin decohering rates,

thus providing an avenue for the control of ASQ regardless of device specifications.

System modeling. We consider a simple model for a nanowire Josephson junction of length $L$ sandwiched between two superconductors with an energy gap $\Delta_{S}$ [see Fig. 1(b)]. In order to obtain a spectrum for the ABSs, we consider the ballistic case with a single occupied subband, characterized by two modes $\left|m_{1,2}\right\rangle$ with different Fermi velocities $v_{1,2}$ and each featuring two possible pseudospin states $|\uparrow\rangle$ and $|\downarrow\rangle[14,21]$. The low energy effective Hamiltonian can be written in this basis as

$$
\begin{aligned}
H_{\mathrm{eff}}= & \sum_{j=1,2} \frac{\Delta_{S}}{1+\lambda_{j}} \frac{\pi-(-1)^{j} \phi \sigma_{z}}{2}\left|m_{j}\right\rangle\left\langle m_{j}\right| \\
& +r \Delta_{S}\left(\left|m_{1}\right\rangle\left\langle m_{2}\right|+\text { H.c. }\right)
\end{aligned}
$$

where $\lambda_{j}=\Delta_{S} L / \hbar v_{j}, \sigma_{z}=|\uparrow\rangle\langle\uparrow|-| \downarrow\rangle\langle\downarrow|$ the Pauli matrix for the pseudospin degree of freedom and we have summarized collisional effects through a reflection term characterized by the coefficient $r$. More details are provided in the Supplemental Material [21]. A simple transformation allows us to write this model as two coupled qubits [21]

$$
\begin{aligned}
H_{\mathrm{eff}} & =\frac{\omega}{2} \tau_{z}-\frac{g}{2} \tau_{z} \sigma_{z}+\frac{v}{2} \sigma_{z}+E_{0} \\
& =\sum_{\substack{k=1,2 \\
s=\uparrow, \downarrow}} \epsilon_{k s}|k s\rangle\langle k s|,
\end{aligned}
$$

where we now define $\tau_{z}$ as the Pauli matrix for the Andreev longitudinal mode degrees of freedom in the basis spanned by states $|1\rangle$ and $|2\rangle$ with an attractive interaction term of strength $g$ that inverts the spin orientation in the upper level and a global energy offset $E_{0}$. Note that the degrees of freedom have become rotated with respect to Eq. (1).

We consider a gate ac driving at frequency $\omega_{g}$ that produces pseudospin-conserving transitions of strength $\Omega_{g}$ and pseudospin-flipping transitions of weaker strength $\alpha \Omega_{g}$

$$
H_{g}(t)=\Omega_{g} \cos \left(\omega_{g} t\right)\left(1+\alpha \sigma_{x}\right) \tau_{x},
$$

and an additional flux ac driving at frequency $\omega_{f}$ that produces pseudospin-conserving transitions of strength $\Omega_{f}$ and no pseudospin-flipping transitions $(\alpha=0)$

$$
H_{f}(t)=\Omega_{f} \cos \left(\omega_{f} t\right) \tau_{x} .
$$

A layout of the available degrees of freedom and couplings is shown in Fig. 2(a).

STIRAP implementation. As shown in Fig. 2(a), we consider a four-level system unlike the typical STIRAP setting of three levels [see Fig. 1(a)]. In order to implement a STIRAP protocol between the initial state $|1 \downarrow\rangle$ and the final state $|1 \uparrow\rangle$, the following Raman resonance condition is imposed:

$$
\Delta \equiv\left(\epsilon_{2 \uparrow}-\epsilon_{1 \downarrow}\right)-\omega_{g}=\left(\epsilon_{2 \uparrow}-\epsilon_{1 \uparrow}\right)-\omega_{f},
$$

such that a rotating wave approximation (RWA) in the relevant interaction picture reveals the transitions

$$
\begin{aligned}
H_{g}^{\prime}+H_{f}^{\prime} \simeq & \frac{\Omega_{f}}{2}|1 \uparrow\rangle\left\langle 2 \uparrow\left|+\frac{\alpha \Omega_{g}}{2}\right| 1 \downarrow\right\rangle\langle 2 \uparrow| \\
& +\frac{\Omega_{g}}{2}|1 \downarrow\rangle\langle 2 \downarrow|+\text { H.c., }
\end{aligned}
$$


(a)

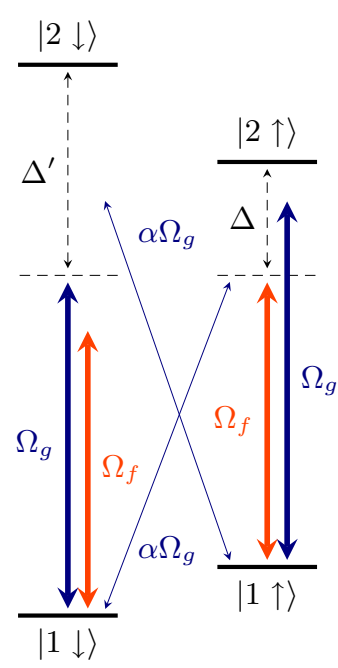

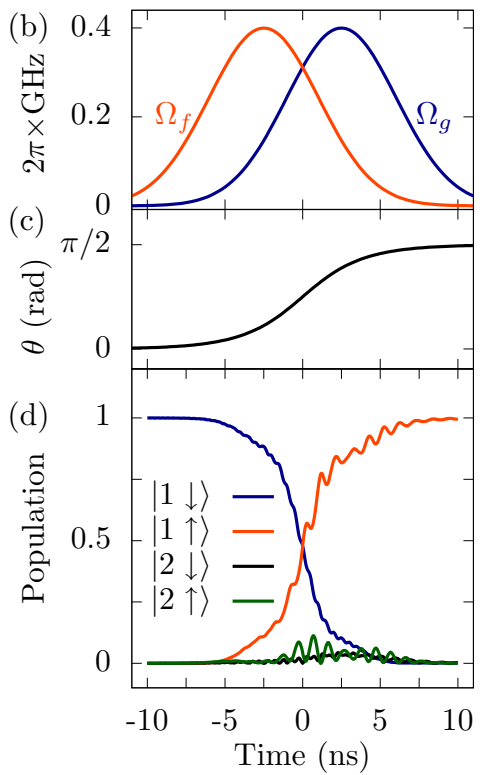

FIG. 2. (a) Schematic level diagram with available couplings generated by gate ac driving (blue) and magnetic flux ac driving (orange). The frequencies $\omega_{g}$ and $\omega_{f}$ are chosen such that the Raman resonance condition Eq. (5) is fulfilled. (b) Rabi frequencies $\Omega_{g}$ and $\Omega_{f}$ as a function of time for $\alpha=1$. Otherwise, the intensity of the gate pulse has to be enhanced by $\alpha^{-1}$ to achieve transfer. (c) Value of $\theta=\arctan \left(\alpha \Omega_{g} / \Omega_{f}\right)$ as a function of time for the pulses in (b). (d) Dynamics of the occupation probabilities of states $|1 \downarrow\rangle,|1 \uparrow\rangle$, $|2 \downarrow\rangle$, and $|2 \uparrow\rangle$ under the effect of the pulses in (b) and for $\alpha=1$, $\Delta=0$, and $\Delta^{\prime}=2 \pi \times 2 \mathrm{GHz}$.

including the wanted $\Lambda$ coupling that satisfies the Raman condition and an unwanted vertical coupling detuned by $\Delta^{\prime}=$ $\left(\epsilon_{2 \downarrow}-\epsilon_{1 \downarrow}\right)-\omega_{g}$. It is important to notice that, beyond these three couplings, the RWA neglects three additional unwanted nonresonant couplings as shown in Fig. 2(a). In particular, the coupling between $|1 \uparrow\rangle$ and $|2 \uparrow\rangle$ of strength $\Omega_{g}$ may be even more detrimental due to its proximity to resonance. These couplings can only be captured in a full time-dependent numerical simulation of the sort performed in this Letter. Their effect is in general suppressed with the choice $\Delta=0$, which is the default choice in the remainder of the discussion unless otherwise stated.

The STIRAP protocol consists of the introduction of a slow modulation of the parameters $\Omega_{f}$ and $\Omega_{g}$,

$$
\begin{aligned}
& \Omega_{f}(t)=\alpha A e^{-\left(\frac{t-t_{f}}{T}\right)^{2}}, \\
& \Omega_{g}(t)=A e^{-\left(\frac{t-t_{g}}{T}\right)^{2}},
\end{aligned}
$$

at times $t_{f}<t_{g}$ with Rabi-frequency amplitudes $\alpha A, A$ and widths $T$. Under the time-dependent transformation of the lower states into bright $|B\rangle$ and dark $|D\rangle$ states defined by

$$
\begin{aligned}
& |B\rangle=\frac{1}{\Omega}|1\rangle\left(\alpha \Omega_{g}|\downarrow\rangle+\Omega_{f}|\uparrow\rangle\right), \\
& |D\rangle=\frac{1}{\Omega}|1\rangle\left(\Omega_{f}|\downarrow\rangle-\alpha \Omega_{g}|\uparrow\rangle\right),
\end{aligned}
$$

with $\Omega=\sqrt{\Omega_{f}^{2}+\alpha^{2} \Omega_{g}^{2}}$ the root mean square Rabi frequency, the effective Hamiltonian simplifies to the form

$$
\begin{aligned}
H(t)= & H_{\text {eff }}^{\prime}+H_{g}^{\prime}+H_{f}^{\prime} \\
\simeq & \left(\Delta|\uparrow\rangle\left\langle\uparrow\left|+\Delta^{\prime}\right| \downarrow\right\rangle\langle\downarrow|\right)|2\rangle\langle 2| \\
& +\frac{\Omega}{2}(|2 \uparrow\rangle\langle B|+\text { H.c. })+i \dot{U} U^{\dagger},
\end{aligned}
$$

where the time-dependent transformation $U=|D\rangle\langle 1 \downarrow|+$ $|B\rangle\left\langle 1 \uparrow\left|+\sum_{\sigma=\downarrow, \uparrow}\right| 2 \sigma\right\rangle\langle 2 \sigma|$ introduces the term

$$
i \dot{U} U^{\dagger}=i \dot{\theta}|B\rangle\langle D|+\text { H.c., }
$$

with $\tan \theta=\alpha \frac{\Omega_{g}}{\Omega_{f}}$ so that $\dot{\theta}=\alpha \frac{\dot{\Omega}_{g} \Omega_{f}-\dot{\Omega}_{f} \Omega_{g}}{\Omega^{2}}$. For very slow (adiabatic) variations of the Rabi frequency, the dark state $|D\rangle$ remains decoupled. The mechanism of STIRAP is straightforward from here. A system is initially prepared in $|1 \downarrow\rangle$, which becomes the dark state under the effect of the first pulse, and can be adiabatically transformed into any superposition with $|1 \uparrow\rangle$ by an appropriate choice of the timing of $\theta$. The condition of adiabaticity can be expressed as

$$
\frac{\dot{\theta}}{\Omega} \ll 1
$$

at all times $t$.

We present an implementation of the protocol in Fig. 2. As an example, following [15] we may assume a spectrum with nondegenerate transitions such that

$$
\begin{aligned}
& \epsilon_{1 \uparrow}-\epsilon_{1 \downarrow}=2 \pi \times 1 \mathrm{GHz}, \\
& \epsilon_{2 \downarrow}-\epsilon_{1 \downarrow}=2 \pi \times 13 \mathrm{GHz}, \\
& \epsilon_{2 \uparrow}-\epsilon_{1 \downarrow}=2 \pi \times 11 \mathrm{GHz} .
\end{aligned}
$$

The choice $\Delta=0$ imposes $\omega_{g}=2 \pi \times 11 \mathrm{GHz}, \omega_{f}=$ $2 \pi \times 10 \mathrm{GHz}$, and $\Delta^{\prime}=2 \pi \times 2 \mathrm{GHz}$. Assuming no suppression of spin-flipping transitions $\alpha=1$, a choice of timings $t_{g}=2 T=-t_{f}=2.5 \mathrm{~ns}$ imposes a minimum Rabi-frequency amplitude $A=2 \pi \times 400 \mathrm{MHz}$ in order for Eq. (11) to be satisfied. The population transfer corresponding to such pulses [Figs. 2(b) and 2(c)] is shown in Fig. 2(d). Note a rather small transfer of population to level $|2 \uparrow\rangle$ due to the presence of the strong nonresonant coupling between $|1 \uparrow\rangle$ and $|2 \uparrow\rangle$ generated by the gate driving. Our parametric choice prevents this effect from affecting the success of STIRAP.

Dissipation and state preparation. As investigated in $[15,20]$, the states have both a limited lifetime and coherence time. In their sample, two pseudospin-conserving decay channels between excited and ground levels are found of rates $\Gamma$ ranging between $250 \mathrm{rad} / \mathrm{ms}$ and $333 \mathrm{rad} / \mathrm{ms}$. Additionally, a pseudospin mixing term of rate $\gamma$ drives the system into a maximal mixture of states $|\downarrow\rangle$ and $|\uparrow\rangle$. The numeric value has been investigated in [15] with an inconclusive range between $\simeq 24.4 \mathrm{rad} / \mathrm{ms}$ and $\simeq 0.4 \mathrm{rad} / \mathrm{ms}$, as discussed in [21]. Additionally, the coherence of the spin and of the Andreev longitudinal modes decays at rates $\gamma_{s}$ and $\gamma_{m}$, respectively, which are both of the order of $100 \mathrm{rad} / \mu \mathrm{s}$ [20]. We model these decoherence effects by means of the master 
(a)
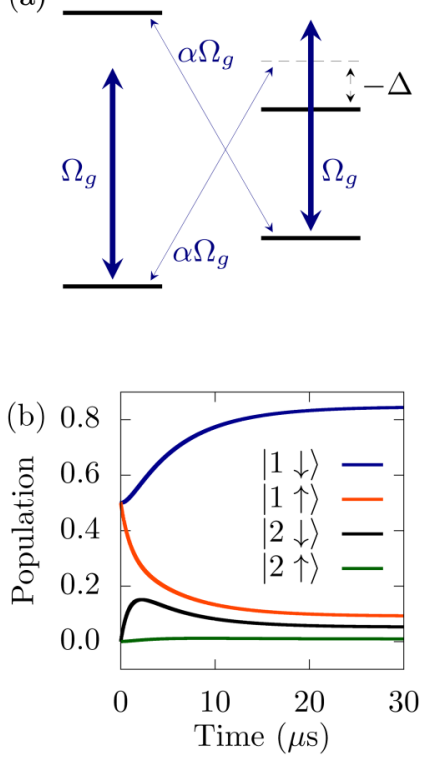

(c)
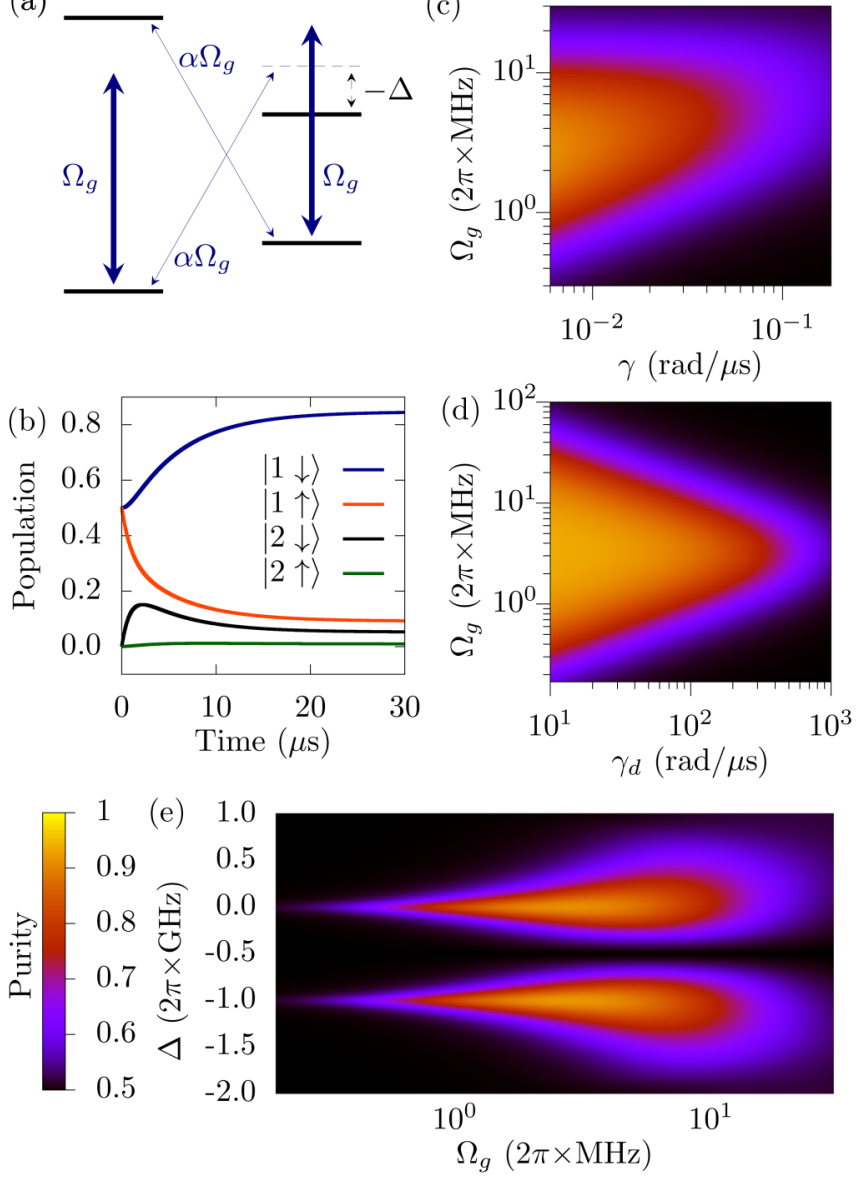

FIG. 3. (a) Resonance condition $\omega_{g}=\epsilon_{2 \downarrow}-\epsilon_{1 \uparrow}$ for the preparation of state $|1 \downarrow\rangle$ with a single gate pulse [note the difference with the arrangement of Fig. 2(a)]. (b) Population dynamics under the effect of a continuous gate pulse of strength $\Omega_{g}=2 \pi \times 3 \mathrm{MHz}$ for $\gamma=2 \times 10^{-2} \mathrm{rad} / \mu \mathrm{s}$ and $\gamma_{d}=10^{2} \mathrm{rad} / \mu \mathrm{s}$. (c) Purity of the steady state of the spin degrees of freedom as a function of the driving Rabi frequency $\Omega_{g}$ and the spin mixing rate $\gamma$ for $\gamma_{d}=10^{2} \mathrm{rad} / \mu \mathrm{s}$. (d) Purity of the steady state of the spin degrees of freedom as a function of the driving Rabi frequency $\Omega_{g}$ and the decoherence rate $\gamma_{d}$ for $\gamma=10^{-2} \mathrm{rad} / \mu \mathrm{s}$. (e) Purity of the steady state of the spin degrees of freedom as a function of the driving Rabi frequency $\Omega_{g}$ and the detuning $\Delta$ for $\gamma=5 \times 10^{-3} \mathrm{rad} / \mu \mathrm{s}$ and $\gamma_{d}=10^{2} \mathrm{rad} / \mu \mathrm{s}$. Purity values may range between 1 (pure state) and 0.5 (fully mixed two-level system).

equation

$$
\begin{aligned}
\frac{d}{d t} \rho= & {[H(t), \rho]+\frac{\gamma_{m}}{2} \mathcal{D}\left[\tau_{z}\right](\rho)+\frac{\gamma_{s}}{2} \mathcal{D}\left[\sigma_{z}\right](\rho) } \\
& +\frac{\Gamma}{2} \mathcal{D}\left[\tau_{-}\right](\rho)+\frac{\gamma}{2} \mathcal{D}\left[\sigma_{-}\right](\rho)+\frac{\gamma}{2} \mathcal{D}\left[\sigma_{+}\right](\rho),
\end{aligned}
$$

where $\tau_{-}=|1\rangle\left\langle 2\left|, \sigma_{-}=\right| \downarrow\right\rangle\langle\uparrow|, \sigma_{+}=\left(\sigma_{-}\right)^{\dagger}$ and with the Lindbladian superoperator $\mathcal{D}[s](\rho)=2 s \rho s^{\dagger}-s^{\dagger} s \rho-\rho s^{\dagger} s$. Note that we assume a similar coherence lifetime of superpositions of $|1\rangle$ and $|2\rangle$ states in line with the findings in [20].

Due to these processes, the system is initially in the fully mixed state $\rho(0)=1 / 2|1\rangle\left\langle 1\left|\sum_{s=\downarrow, \uparrow}\right| s\right\rangle\langle s|$, which imposes the need to prepare the sample in a state of low entropy for any control protocol. Fortunately, the gate pulse in the STIRAP arrangement [Fig. 2(a)] automatically produces an

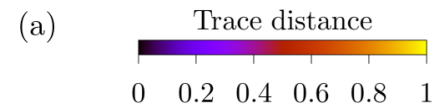

(b) Population transfer

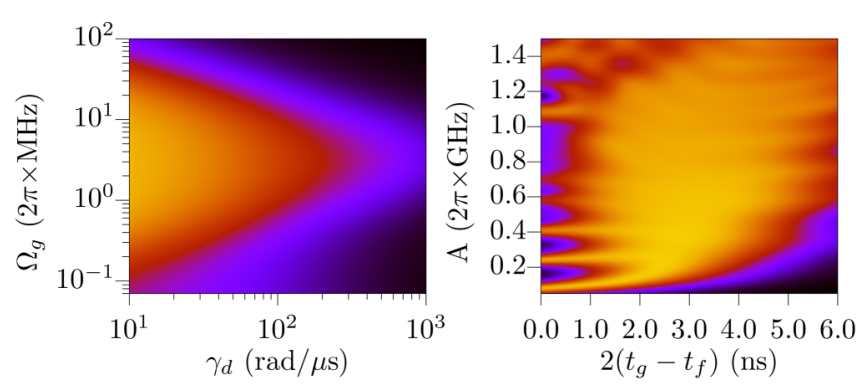

FIG. 4. (a) Trace distance between the spin state before and after the STIRAP protocol as a function of the Rabi frequency $\Omega_{g}$ of the preparation pulse and the decoherence rate $\gamma_{d}$ for $\gamma=10^{-2} \mathrm{rad} / \mu \mathrm{s}$. STIRAP pulses as in Fig. 2(b). (b) Population transferred from state $|1 \downarrow\rangle$ to state $|1 \uparrow\rangle$ after application of the STIRAP protocol as a function of the Rabi-frequency amplitude $A$ and the pulse separation $2\left(t_{g}-t_{f}\right)$ for $T=5 \mathrm{~ns}$, a spin mixing rate $\gamma=10^{-2} \mathrm{rad} / \mu \mathrm{s}$, and a decoherence rate $\gamma_{d}=10^{2} \mathrm{rad} / \mu \mathrm{s}$.

optical pumping effect that depletes the state $|1 \downarrow\rangle$, similar to the case shown in [20]. For an appropriate choice of $\Delta$ [see Fig. 3(a)], the state $|1 \uparrow\rangle$ may be depleted instead [Fig. 3(b)]. This is due to the resonance with the $|1 \uparrow\rangle-|2 \downarrow\rangle$ transition, which pumps population from $|\uparrow\rangle$ to $|\downarrow\rangle$ that then preferentially decays to $|1 \downarrow\rangle$.

The effect of the polarizing pulse may be described in terms of the rate equation [21]

$$
\frac{d}{d t}\left(\begin{array}{l}
p_{\downarrow} \\
p_{\uparrow}
\end{array}\right)=\left(\begin{array}{cc}
-\gamma_{\uparrow}-\gamma & \gamma_{\downarrow}+\gamma \\
\gamma_{\uparrow}+\gamma & -\gamma_{\downarrow}-\gamma
\end{array}\right)\left(\begin{array}{l}
p_{\downarrow} \\
p_{\uparrow}
\end{array}\right) .
$$

For an optimal choice of parameters, effective decay rates $\gamma_{\uparrow} \simeq 0$ and $\gamma_{\downarrow} \simeq \Gamma$ may be reached, so that the population inversion $\Gamma /(2 \gamma+\Gamma)$ is achieved. In our case, this approaches 1 since $\gamma \ll \Gamma$ [see Fig. 3(b)], and this becomes a crucial criterion to select the parametric region for the implementation of STIRAP, see Fig. 3(c). The choice of $\Omega_{g}$ is additionally affected by the decoherence rate $\gamma_{d}$, as shown in Fig. 3(d). Finally, it is equally possible to address the detuning value $\Delta=0$ in order to prepare the state $|1 \uparrow\rangle$ with similar efficiency, see Fig. 3(e).

Global efficiency of the protocol. The final success of state transfer from $|1 \downarrow\rangle$ into $|1 \uparrow\rangle$ after the polarization pulse into $|1 \downarrow\rangle$ and the STIRAP protocol can be measured in terms of the trace distance $\operatorname{Tr}\left[\sqrt{\left(\rho_{0}-\rho_{t}\right)^{2}}\right]$ between the spin density matrices before $\rho_{0}$ and after $\rho_{t}$ the STIRAP protocol. Its maximum is 1 for orthogonal states, while it vanishes for equal states. For a two-level system, it represents an appropriate measure of the success of the transfer for mixed states. This is shown in Fig. 4(a). The transfer is successful for a large range of preparation pulse strengths and decoherence rates, but it is mostly dependent on the purity of the initial state, as one can deduce from comparing Figs. 4(a) and 3(d). At the same time, variations in the overall Rabi frequency $A$ and the separation between pulses $t_{g}-t_{f}$ do not substantially affect the final transfer efficiency, as shown in Fig. 4(b). On the contrary, application of continuous Raman pulses (corresponding to the left edge of the same figure) is highly sensitive to pulse 
strength, whereas STIRAP is robust over a large region. This makes this proposal suitable for varying design parameters.

Conclusions. We have proposed a means to the manipulation of the pseudospin degrees of freedom of Andreev bound states that includes a polarization pulse and subsequent coherent manipulation with the STIRAP technique. We have shown that the protocol could be efficient even for the stringent conditions of actual present experiments in which coherence times are rather short and is in line with current efforts to develop an ASQ by several experimental groups.

Acknowledgments. The authors acknowledge C. Urbina for his useful comments. A.L.Y. and J.C. acknowledge European
Union's Horizon 2020 research and innovation programme for financial support through Grant No. 828948 (AndQC) and QuantERA Project No. 127900 (SuperTOP). J.C. acknowledges the support from Ministerio de Ciencia, Innovación y Universidades (Spain) ("Beatriz Galindo" Fellowship BEAGAL18/00081). A.L.Y. acknowledges support by Spanish MICINN through Grant No. FIS2017-84860-R and through the María de Maeztu Programme for Units of Excellence in R\&D (Grant No. MDM-2014-0377). M.H. and V.F. acknowledge support by the US Office of Naval Research (Grant No. N00014-16-1-2270) and by the US Army Research Office (Grants No. W911NF-18-1-0020 and No. W911NF-18-10212).
[1] F. Arute et al., Quantum supremacy using a programmable superconducting processor, Nature (London) 574, 505 (2019).

[2] T. Karzig, C. Knapp, R. M. Lutchyn, P. Bonderson, M. B. Hastings, C. Nayak, J. Alicea, K. Flensberg, S. Plugge, Y. Oreg, C. M. Marcus, and M. H. Freedman, Scalable designs for quasiparticle-poisoning-protected topological quantum computation with Majorana zero modes, Phys. Rev. B 95, 235305 (2017).

[3] M. A. Despósito and A. L. Yeyati, Controlled dephasing of Andreev states in superconducting quantum point contacts, Phys. Rev. B 64, 140511(R) (2001).

[4] A. Zazunov, V. S. Shumeiko, E. N. Bratus, J. Lantz, and G. Wendin, Andreev Level Qubit, Phys. Rev. Lett. 90, 087003 (2003).

[5] C. Janvier, L. Tosi, L. Bretheau, Ç. Ö. Girit, M. Stern, P. Bertet, P. Joyez, D. Vion, D. Esteve, M. F. Goffman, H. Pothier, and C. Urbina, Coherent manipulation of Andreev states in superconducting atomic contacts, Science 349, 1199 (2015).

[6] N. M. Chtchelkatchev and Y. V. Nazarov, Andreev Quantum Dots for Spin Manipulation, Phys. Rev. Lett. 90, 226806 (2003).

[7] C. Padurariu and Y. V. Nazarov, Theoretical proposal for superconducting spin qubits, Phys. Rev. B 81, 144519 (2010).

[8] S. Park and A. L. Yeyati, Andreev spin qubits in multichannel Rashba nanowires, Phys. Rev. B 96, 125416 (2017).

[9] E. Prada, P. San-Jose, M. W. A. de Moor, A. Geresdi, E. J. H. Lee, J. Klinovaja, D. Loss, J. Nygård, R. Aguado, and L. P. Kouwenhoven, From Andreev to Majorana bound states in hybrid superconductor-semiconductor nanowires, Nat. Rev. Phys. 2, 575 (2020).

[10] R. M. Lutchyn, J. D. Sau, and S. Das Sarma, Majorana Fermions and a Topological Phase Transition in Semiconductor-Superconductor Heterostructures, Phys. Rev. Lett. 105, 077001 (2010).

[11] Y. Oreg, G. Refael, and F. von Oppen, Helical Liquids and Majorana Bound States in Quantum Wires, Phys. Rev. Lett. 105, 177002 (2010).
[12] D. J. van Woerkom, A. Proutski, B. van Heck, D. Bouman, J. I. Väyrynen, L. I. Glazman, P. Krogstrup, J. Nygård, L. P. Kouwenhoven, and A. Geresdi, Microwave spectroscopy of spinful Andreev bound states in ballistic semiconductor Josephson junctions, Nat. Phys. 13, 876 (2017).

[13] M. Hays, G. de Lange, K. Serniak, D. J. van Woerkom, D. Bouman, P. Krogstrup, J. Nygård, A. Geresdi, and M. H. Devoret, Direct Microwave Measurement of Andreev-BoundState Dynamics in a Semiconductor-Nanowire Josephson Junction, Phys. Rev. Lett. 121, 047001 (2018).

[14] L. Tosi, C. Metzger, M. F. Goffman, C. Urbina, H. Pothier, S. Park, A. L. Yeyati, J. Nygård, and P. Krogstrup, Spin-Orbit Splitting of Andreev States Revealed by Microwave Spectroscopy, Phys. Rev. X 9, 011010 (2019).

[15] M. Hays, V. Fatemi, K. Serniak, D. Bouman, S. Diamond, G. de Lange, P. Krogstrup, J. Nygård, A. Geresdi, and M. H. Devoret, Continuous monitoring of a trapped, superconducting spin, Nat. Phys. 16, 1103 (2020).

[16] K. Bergmann et al., Roadmap on STIRAP applications, J. Phys. B: At. Mol. Opt. Phys. 52, 202001 (2019).

[17] J. Siewert, T. Brandes, and G. Falci, Adiabatic passage with superconducting nanocircuits, Opt. Commun. 264, 435 (2006).

[18] K. S. Kumar, A. Vepsäläinen, S. Danilin, and G. S. Paraoanu, Stimulated Raman adiabatic passage in a three-level superconducting circuit, Nat. Commun. 7, 10628 (2016).

[19] C. Metzger, S. Park, L. Tosi, C. Janvier, A. A. Reynoso, M. F. Goffman, C. Urbina, A. L. Yeyati, and H. Pothier, Circuit-QED with phase-biased Josephson weak links, Phys. Rev. Research 3, 013036 (2021).

[20] M. Hays, V. Fatemi, D. Bouman, J. Cerrillo, S. Diamond, K. Serniak, T. Connolly, P. Krogstrup, J. Nygård, A. L. Yeyati, A. Geresdi, and M. H. Devoret, Coherent manipulation of an Andreev spin qubit, arXiv:2101.06701.

[21] See Supplemental Material at http://link.aps.org/supplemental/ 10.1103/PhysRevResearch.3.L022012 for a derivation of the effective model, the analysis of the spin-mixing rate values, and a derivation of the rate equation for spin polarization. 